\title{
3.9GHz Accelerating Cavity Field Detection Hardware for the Free-Electron Laser
}

Abstract: The 3rd harmonic system installed in the injector at FLASH (Free-Electron Laser at Hamburg) accelerator allows to achieve ultra short electron bunches with higher peak current, which pushed the limits of high brilliance laser light generation into the so-called water window. The 3rd harmonic system operates at $3.9 \mathrm{GHz}$ and requires a very precise phase and amplitude control of the accelerating field below 10fs, which is done by the LowLevel RF system. One of the key component is the RF field detector that converts the detected cavity field signal to an intermediate frequency for digital sampling. The paper presents an overview of the system, particularly the low phase noise reference generator, local oscillator and multichannel down-converter. System scheme and performance are demonstrated.

\section{Overview of the 3.9 GHz 3rd harmonic Low-Level-RF system at FLASH}

The 3rd harmonic system operating at 3.9GHz must be phase-locked to the accelerating modules operating at $1.3 \mathrm{GHz}$ respectively to the overall master oscillator reference. Additionally for high field detection accuracy it is required that al signals in the 3rd harmonic system have constant phase-relations to the $3.9 \mathrm{GHz}$ reference.

Phase noise and drifts of all subsystems, especially the $3.9 \mathrm{GHz}$ reference (REF) $3.954 \mathrm{GHz}$ local oscillator (LO), $81 \mathrm{MHz}$ clock and the down-converter itself limit the accuracy of cavity field control.

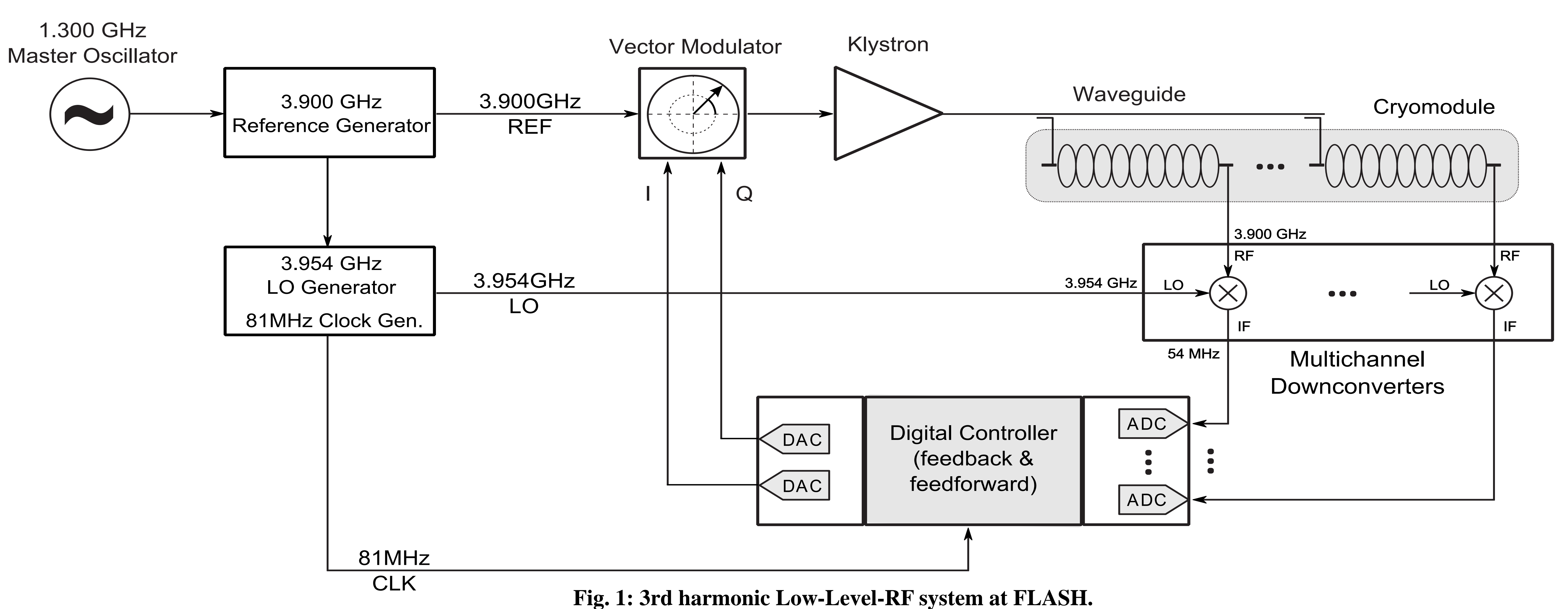

\subsection{GHz Reference Generation}

The phase noise and the long term drifts are minimized simultaneously by the choice of the proper $\underset{\text { trom } \mathrm{MO}}{130 \mathrm{MH}}$ cut-off frequencies for both detectors.

To achieve low phase drifts the low-pass filter has an integrating section.

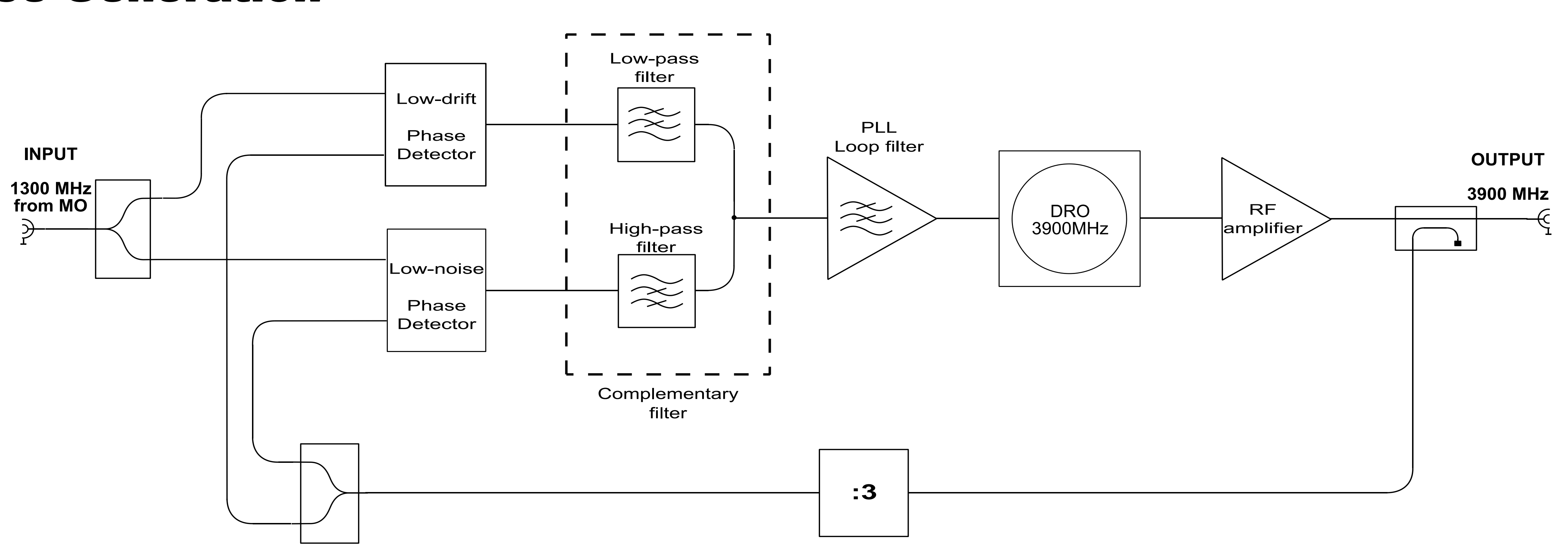

Fig. 2: Block diagram of the $3.9 \mathrm{GHz}$ reference generator:

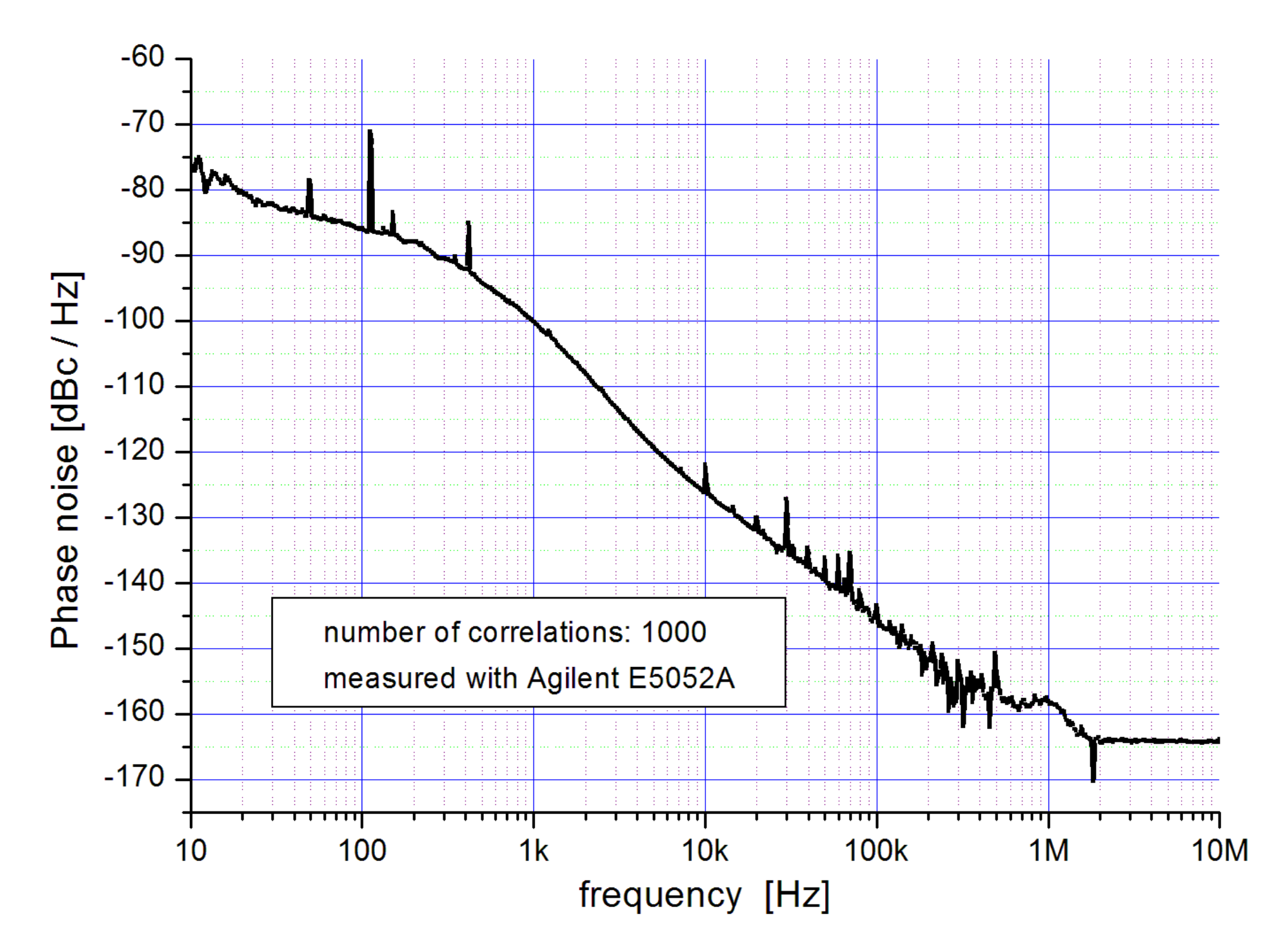

Fig. 3: Phase noise of the $3.9 \mathrm{GHz}$ reference generator.

\subsection{GHz LO and 81 MHz Clock Generation}

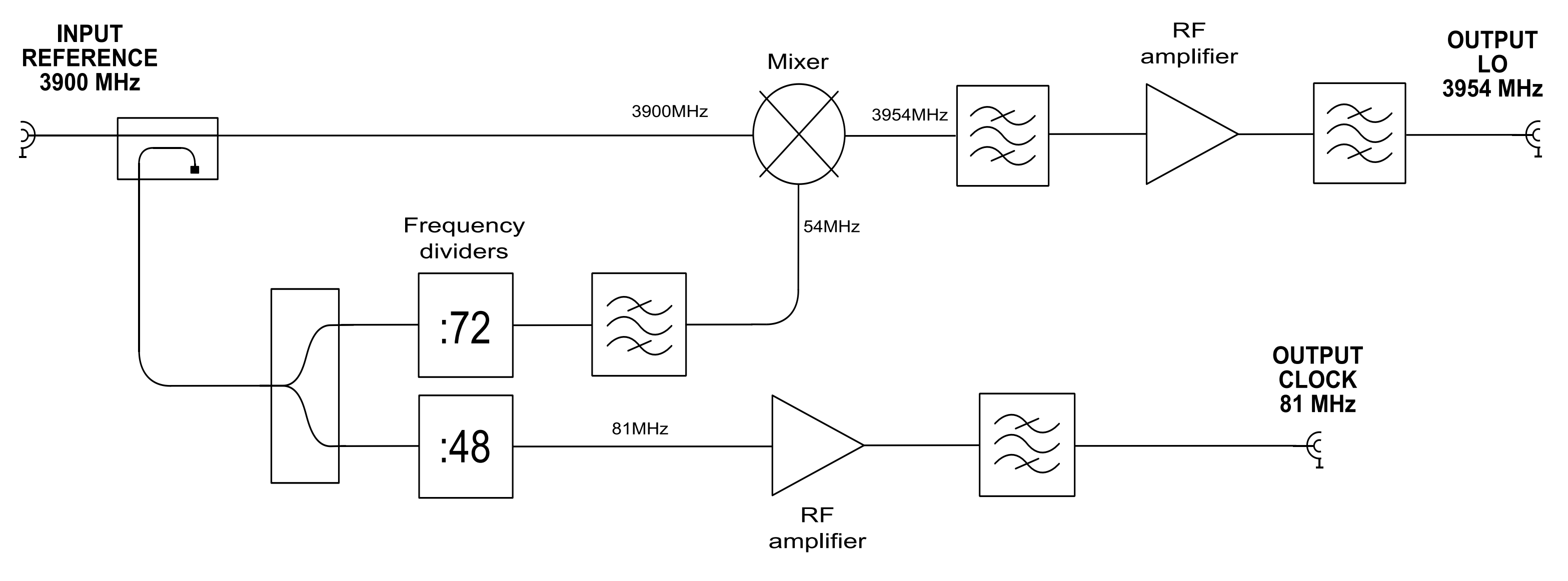

Fig. 4: Block diagram of the 3.954GHz local oscillator and clock generation
Multichannel Down-Converter

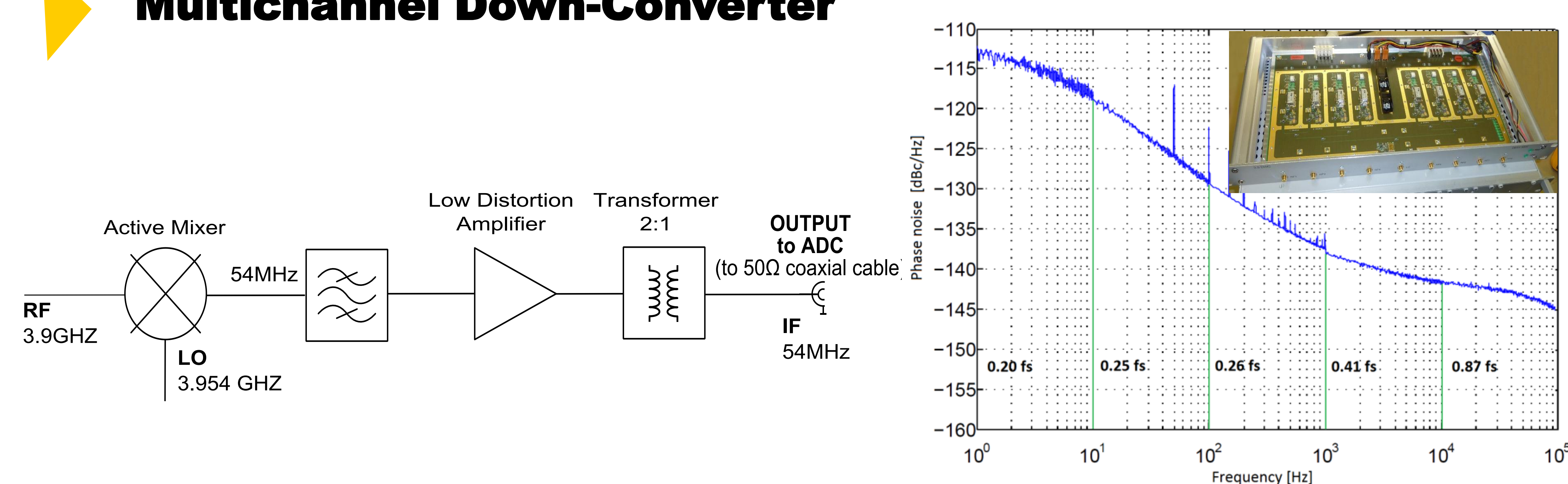

Fig. 5:Simplified schematic of the down-converter cell.

\section{Summary}

The system was installed and tested at the 3rd harmonic system at the FLASH accelerator. The measured short-term phase stability of the system was determined to $3.3 \mathrm{fs}$, respectively $4.6 \mathrm{mdeg}$ at $3.9 \mathrm{GHz}$, which clearly fulfills the cavity field stability requirements of $<10^{-5}$ in amplitude and $<\mathrm{0.01}^{\circ}$ in phase. The system was installed in 2010 and works successfully without performance degradation.

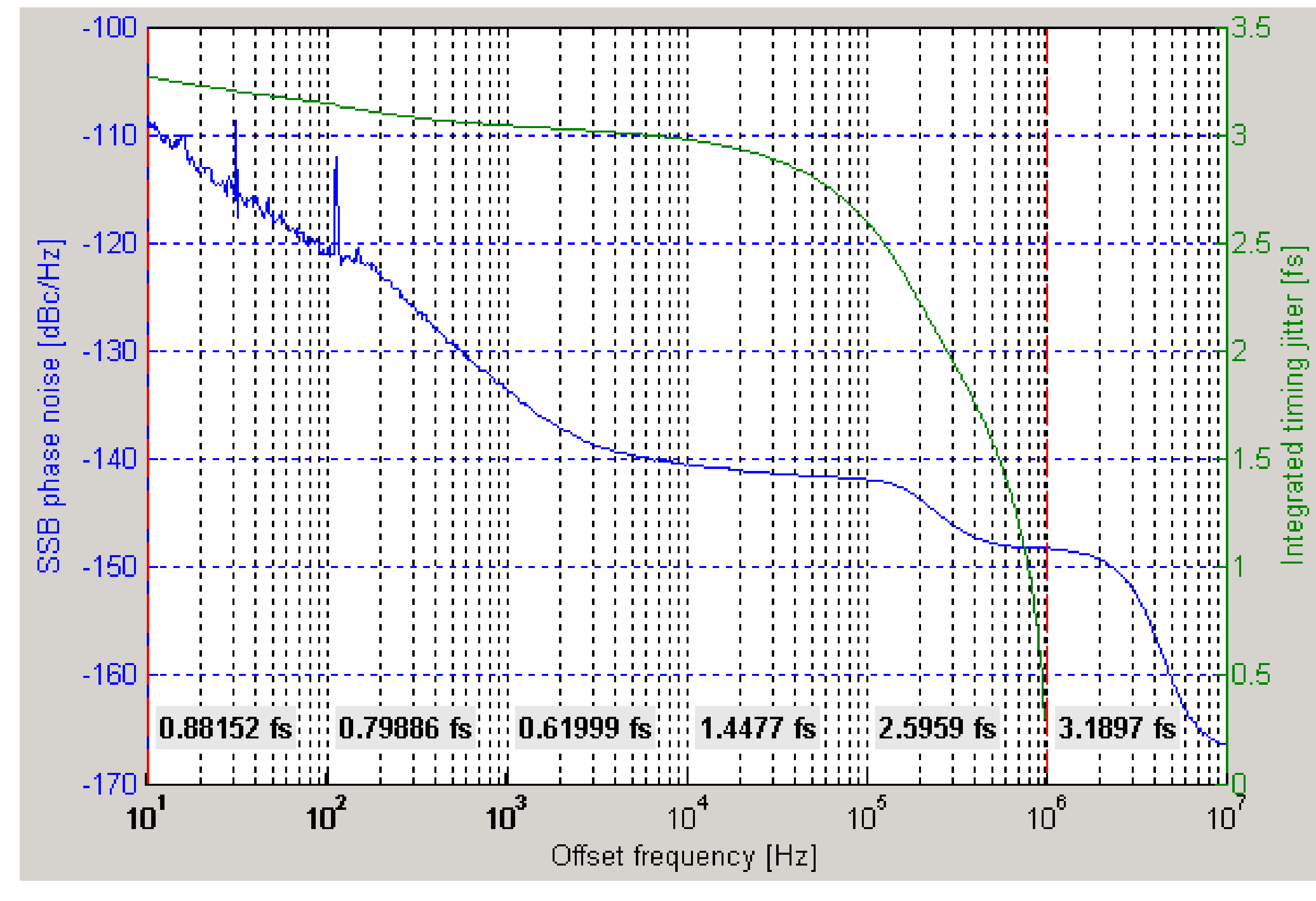

Fig. 7: Phase noise of the 54MHz IF signal of the down-converter output. The integrated jitter was 3.3fs
Jan Piekarski, Mateusz Żukociński, Krzysztof Czuba Institute of Electronic Systems, Warsaw University of Technology

E-mail: j.piekarski@stud.elka.pw.edu.pl
Matthias Hoffmann, Frank Ludwig, Holger Schlarb Deutsches Elektronen-Synchrotron , Hamburg 